\title{
Modeling of Turbulence Effects on Liquid Jet Atomization and Breakup
}

\author{
Huu P. Trinh* \\ NASA-Marshall Space Flight Center. MSFC. AL 35812 \\ C. P. Chen ${ }^{\dagger}$ \\ University of Alabama in Huntsville, Huntsville, AL 35899
}

\begin{abstract}
Recent experimental investigations and physical modeling studies have indicated that turbulence behaviors within a liquid jet have considerable effects on the atomization process. This study aims to model the turbulence effect in the atomization process of a cylindrical liquid jet. Two widely used models, the KelvinHelmholtz (KH) instability of Reitz (blob model) and the Taylor-Analogy-Breakup (TAB) secondary droplet breakup by O'Rourke et aL, are further extended to include turbulence effects. In the primary breakup model, the level of the turbulence effect on the liquid breakup depends on the characteristic scales and the initial flow conditions. For the secondary breakup, an additional turbulence force acted on parent drops is modeled and integrated into the TAB governing equation. The drop size formed from this breakup regime is estimated based on the energy balance before and after the breakup occurrence. This paper describes theoretical development of the current models, called " $T$-blob" and " $T-T A B$ ", for primary and secondary breakup respectively. Several assessment studies are also presented in this paper.
\end{abstract}

\section{Nomenclature}

a Radius of blob or parent drop

$A_{1} \quad$ Constant (0.188)

$B_{0} \quad$ Constant (0.61)

$B_{1} \quad$ Constant (10.0)

$\mathrm{C}_{2} \quad$ Constant $\left(\mathrm{B}_{\mathrm{o}} / 3.726 \mathrm{~B}_{1}\right)$

$\mathrm{C}_{\mathrm{b}} \quad$ Constant $(1 / 2)$

$\mathrm{C}_{d} \quad$ Discharge coefficient of injection nozzle; constant used in equation (18)

$C_{D}$. Drag coefficient of a deformed droplet used in equation (27)

$\mathrm{C}_{\mathrm{F}} \quad$ Constant (1/3)

$\mathrm{C}_{\text {jet }} \quad$ Constant $(10)$

$\mathrm{C}_{\mathrm{k}} \quad$ Constant (8)

$\mathrm{C}_{\mathrm{t}} \quad$ Empirical constant involving turbulence force

$c_{1} \quad$ Weighting parameter associated with turbulence motion

$c_{w} \quad$ Weighting parameter associated with surface motion

$c_{\mu} \quad$ Turbulence constant (0.09)

$c_{\varepsilon} \quad$ Turbulence constant (1.92)

$\mathrm{d}_{\max } \quad$ Maximum possible diameter of product drop

$\mathrm{d}_{\min } \quad$ Minmum possible diameter of product drop

D Diameter of injection nozzle

E Term associated with energy

F Aerodynamic force acting on the drop

$F_{1} \quad$ Force associated with turbulence motion in a liquid droplet

$k$ Turbulence kinetic energy per unit mas in the $k-\varepsilon$ model

\footnotetext{
- Aerospace Engineer, Combustion Device Branch/ER32.

${ }^{\dagger}$ Professor, Department of Chemical and Materials Engineering, AlAA Senior Member.
} 


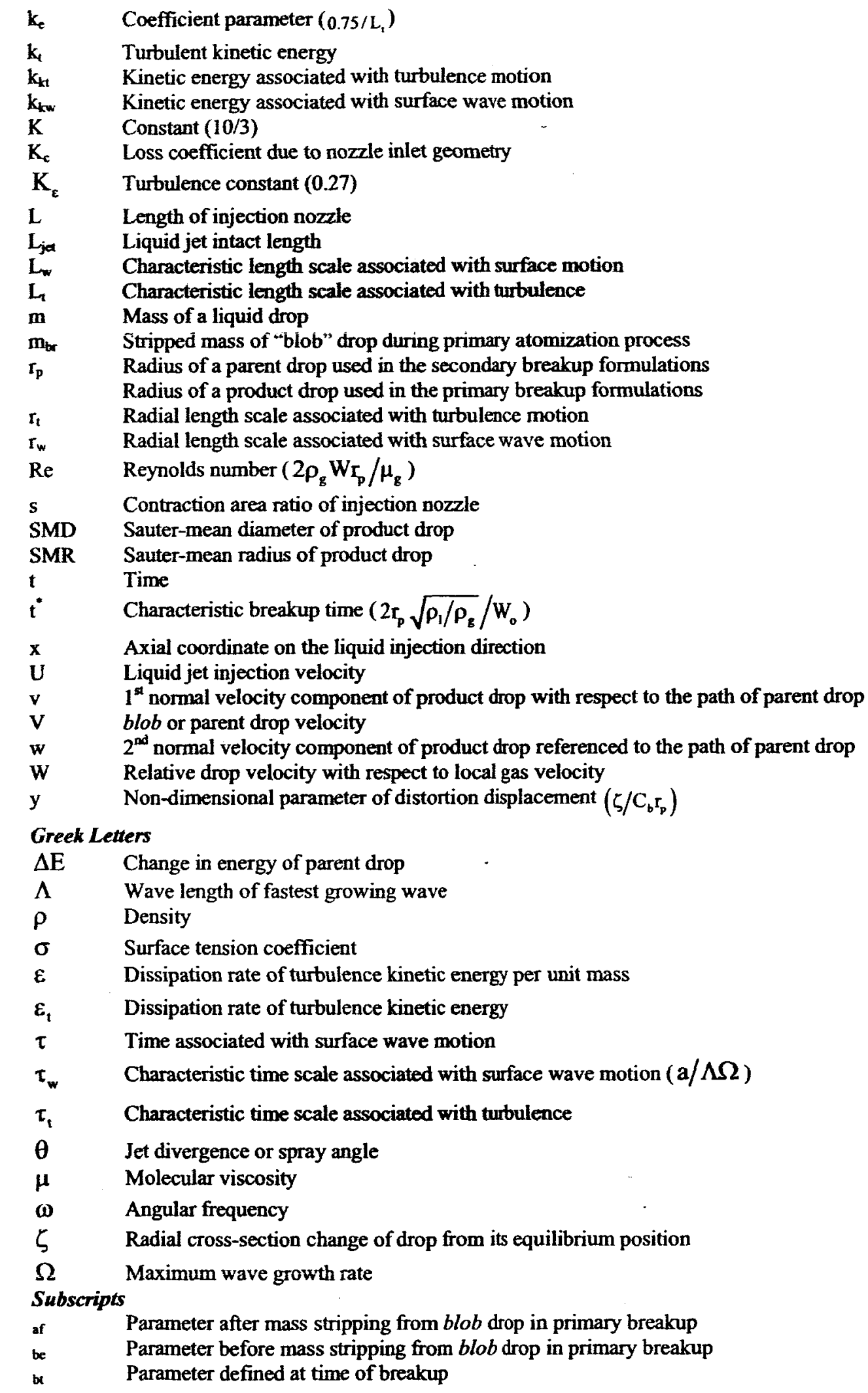




\begin{tabular}{|c|c|}
\hline 0 & Initial value \\
\hline $\mathbf{g}$ & Value of ambient gas \\
\hline$k$ & Parameter associated with motion kinetic energy \\
\hline 1 & Value of liquid phase \\
\hline()$_{\text {par }}$ & Parameter associated with parent drop \\
\hline()$_{\text {prod }}$ & Parameter associated with product drop \\
\hline t & Parameter associated with turbulence \\
\hline tur & $\begin{array}{l}\text { Parameter associated with turbulent kinetic energy } \\
\text { Parameter associated with surface tension energy }\end{array}$ \\
\hline $\mathbf{w}$ & Parameter associated with surface wave motion \\
\hline \multicolumn{2}{|c|}{ Superscripts } \\
\hline o & Initial value \\
\hline & $\begin{array}{l}\text { First derivative with respect to time } \\
\text { Secondary derivative with respect to time }\end{array}$ \\
\hline
\end{tabular}

\section{INTRODUCTION}

$\mathrm{T}$ HE transformation of a liquid body into droplet sprays in a gaseous surrounding is of great importance in many industrial processes. In the liquid fuel combustion systems, such as rocket engines, diesel engines, gas turbines, and industrial furnaces, the combustion efficiency and chemical reaction behavior are primarily dependent on the effectiveness of the liquid body broken up into sprays. Smaller drop sizes generated from the spray devices increase the specific surface area of the fuel and thereby achieve high rates of mixing and evaporation. On the other hand, when fuel is mixed and then reacts rapidly near the injector, the injector exit surface could be overheated inadvertently. These phenomena have been observed in many liquid rocket engines during their hardware development phase. Therefore, understanding and adequately predicting this physical breakup process leads to designing better spray devices for these various applications.

The mechanisms of atomizing the liquid jet are complex. The jet inertial and aerodynamic forces along with the surface tension certainly play a role in the breakup process. In addition, the turbulence behavior inside the liquid jet also contributes to the jet disintegration. Often the geometrical sharpness of the injection nozzle inlet, along with appropriate flow conditions, can create cavitations inside the nozzle. The collapse of this cavitation can generate a flow fluctuation, leading to a more aggressive disintegration of the liquid jet. Previous studies [1-4] showed that the breakup length of the liquid core was significantly shortened when the liquid jet changed from laminar to turbulent flow. More recent studies of relatively large-size liquid jets [5-8] provided droplet data such as size, velocity, fluctuating quantities and reported a more complete mechanistic approach for the primary atomization regime, and indicated that the turbulence developed inside the jet column, starting at the nozzle exit, remained dominant and became a main contributor in the spray development. The authors believed that this turbulence characteristic played a primary role on the liquid stripping near the injector exit. Based on the data collected from their experiments, the authors were able to establish correlations between the turbulence fluctuation quantities and the breakup drop size and the breakup length of the liquid jet.

In the modeling arena, as to the convenience of implementing into computational fluid dynamic (CFD) methods and the wide utilization by analysts, the two most noticeable atomization models are the KH instability model of Reitz [9] and the TAB of O'Rourke et al. [10]. Reitz derived the KH model, also known as the "blob" model or "stripping rate" model, which described the primary breakup entirely in terms of the surface wave perturbation, in which the viscosity, surface tension, and aerodynamic forces were the contributing factors. On the other hand, the TAB model was based on an analogy between an oscillating, distorting droplet and a spring-mass system. In this model the drop distortion was driven by the force interaction among the external aerodynamic, surface tension, and viscous damping of the droplet liquid. The TAB model is suitable for predicting the secondary breakup regime. Recently, Tanner [11] examined data from liquid jet breakup experiments at high pressure conditions and found that an intact liquid core is either broken into various liquid shapes or drop sizes shortly after the injection exit port. 
Hence, the author extended the TAB model and developed a so called enhanced TAB (ETAB) model, to include the primary breakup regime. In his recent Cascade Atomization Breakup (CAB) model for high-pressure liquid jets, Tanner [12] also accounted for the droplet-surface stripping near the injector exit in his ETAB model with a power law drop-size distribution. Though these models provide reasonable predictions of the liquid atomization in some aspects, they do not account for the liquid turbulence motion observed in certain sprays as previously described. In a study of the primary diesel fuel atomization, Nishimura et al. [13] developed a phenomenological cavitation model in which the primary breakup is governed by the turbulence mechanism. The turbulence energy is formed by the bubble collapse and the fluid turbulence motion. To consider the nozzle exit turbulence conditions in the modeling of diesel sprays, Huh et al. [14] proposed a scheme taking into account two independent mechanisms, wave growth and turbulence in the atomization process. The turbulence is characterized partially by the injection nozzle geometry while the wave growth is derived from the $\mathrm{KH}$ instability theory. With this attempt a logical framework of coupling the flow inside the injection nozzle to the jet breakup can be achieved. The rationale for this approach is based on an order-of-magnitude analysis of the flow dynamic breakup mechanisms and the ones associated with the turbulence. This analysis concludes that the gas inertia force and turbulent stress are the two main forces in the atomization of typical diesel engine injectors. In this model, the breakup rate for the parent drops is set to be proportional to the length scale to time scale ratio. It is hypothesized that the turbulence length scale is primarily dominant in the primary breakup while the wave length scale is important for the secondary droplet breakup. On the other hand, the time scale is a linear sum of the turbulence and wave growth time scales.

The aforementioned models were formulated with semi-empiricism. The associated coefficients had to be determined with reference to experimental data. Another class of atomization models was derived based on the conservation laws of mass, momentum, and energy. Several efforts of using sophisticated numerical approaches have been performed in modeling the detailed turbulent flow fields in the liquid and gas during the atomization process. Klein $e t$ al. [15] and De Villiers $e t$ al. [16] computed the two-phase flows using the large eddy simulation (LES) method. For the first time, De Villiers $e t$ al. were able to apply such a numerical technique to resolve the jet atomization under more realistic operating conditions found in Diesel engines. Leboissetier et al. [17] also attempted to simulate a liquid spray with a multi-dimensional pseudo-direct numerical simulation (DNS) method. These methods have a real potential of providing a complete physical description of the liquid jet breakup with minimum assumptions; however, they require small computational time steps and fine grids across the entire jet domain for their simulations. Especially the LES and DNS techniques may need submicron spatial elements in size and pico-second in time steps, to properly predict the atomizing sprays at the high-velocity injection conditions. Consequently, grid mesh size and the considered physical domain must be taken care of so that the computational time and memory storage requirements can be manageable. At the present time they are still too expensive and generally impractical in terms of computational time and power requirements for engineering calculation applications. Hence, the engineering analysis and design of the liquid spray devices still must rely on phenomenological engineering models.

As indicated earlier, the results of experiments suggest that the turbulence motion within the liquid may alter the intact jet core as well as the production of drops, which have a different size in comparison with the droplet breakup without the turbulence consideration. Thus, an accurate modeling of such turbulence effects on the atomization process would significantly enhance the prediction capability of existing physical models. The purpose of this research aims to model the turbulence effects on the primary and secondary atomization processes. In the course of this study, terms accounting for the turbulence motion within a liquid are developed, based on a phenomenological point of view. These terms are appropriately supplemented to the two classical atomization models: HK primary breakup [9] and TAB secondary breakup [10], respectively. In the primary atomization model, the level of the turbulence effects on the mass stripping of the blob drops and on the product drop size is represented by the characteristic time and length scales and the initial kinetic energy. This treatment offers contributions of individual physical phenomena on the liquid breakup. For the secondary breakup an additional turbulence effect, acted on the parent drops, is modeled and integrated into the TAB governing equation. This turbulence term is referenced to the dissipation rate of the turbulent kinetic energy and the deformation rate of the parent drop distortion displacement. The results of the new models and the existing ones are compared and appraised using available experimental data. 


\section{MODEL DEVELOPMENT}

\section{A. T-blob Model}

The original formulation of the primary breakup blob model is based on the stability analysis performed by Reitz [9]. The main contribution of this research effort is to incorporate the turbulence effect in modeling of the liquid jet breakup. Terms and parameters associated with the turbulence behavior are derived and implemented appropriately to the existing blob model. Hence, it is appropriate to denote the present model as " $T$-blob" model. In order to account for the both effects of the surface wave perturbation and the turbulence motion, the resulting formulation would include the combination of these two phenomena in the breakup process. The length scale and time scale associated with the primary breakup are comprised of the ones described in the blob model that represents the surface wave instability and of the turbulence behavior following the approach of Huh et al. [14]. To derive the new model, it is proposed that the rate of change in the parent drop radius has an extra term associated with turbulence effect in the form of

$$
\frac{d a}{d t}=-\left[\frac{a}{\tau}-C_{a}\left(\frac{L_{w}}{\tau_{w}}-\frac{L_{t}}{\tau_{t}}\right)\right] \text {, when } r_{p} \ll a \text {, }
$$

where

$$
\begin{aligned}
& \mathrm{L}_{\mathrm{w}}=\Lambda \\
& \tau_{\mathrm{w}}=\mathrm{a} / \Lambda \Omega \\
& \tau=3.726 \mathrm{~B}_{1} \mathrm{a} / \Lambda \Omega \\
& \mathrm{C}_{\mathrm{a}}=\frac{\mathrm{B}_{0}}{3.726 \mathrm{~B}_{1}}
\end{aligned}
$$

The characteristic length scale $\mathrm{L}_{\mathrm{w}}$ and time scale $\tau_{w}$ are associated with the droplet surface wave instability. These two scales along $\tau$ and $\mathrm{C}_{\mathrm{a}}$ are formulated from the "blob" model. The turbulence characteristic length scale $\mathrm{L}_{1}$ and time scales $\tau_{t}$ can be derived using the analytical solution of the $k-\varepsilon$ turbulence model.

$$
\begin{aligned}
& \tau_{t}=\tau_{t}^{0}+0.0828 t \\
& L_{t}=L_{t}^{0}\left(1+\frac{0.0828 t}{\tau_{t}^{0}}\right)^{0.457} .
\end{aligned}
$$

The time $t$ is counted from the time at which the parent drop leaves the injection nozzle exit. The initial turbulence length scale $\mathrm{L}_{\mathrm{t}}^{0}$ and time scale $\tau_{\mathrm{t}}^{0}$ are evaluated from the initial turbulent kinetic energy $\mathrm{k}_{t}^{0}$ and its corresponding dissipation rate $\varepsilon_{t}^{0}$ at the injector exit.

$$
\begin{aligned}
& \mathrm{L}_{\mathrm{t}}^{0}=\mathrm{c}_{\mu} \frac{\left(\mathbf{k}_{\mathrm{t}}^{0}\right)^{3 / 2}}{\varepsilon_{\mathrm{t}}^{0}} \\
& \tau_{\mathrm{t}}^{0}=\mathrm{c}_{\mu} \frac{\mathbf{k}_{\mathrm{t}}^{0}}{\varepsilon_{\mathrm{t}}^{0}},
\end{aligned}
$$

where

$$
c_{\mu}=0.09 \text {. }
$$

The initial turbulent kinetic energy $\mathbf{k}_{t}^{0}$ and its corresponding dissipation rate $\varepsilon_{\mathrm{t}}^{0}$ can be approximated as

$$
\mathrm{k}_{\mathrm{t}}^{0}=\frac{\mathrm{U}^{2}}{8 \mathrm{~L} / \mathrm{D}}\left[\frac{1}{\mathrm{C}_{\mathrm{d}}^{2}}-\mathrm{K}_{\mathrm{c}}-\left(1-\mathrm{s}^{2}\right)\right]
$$




$$
\varepsilon_{\mathrm{t}}^{0}=\mathrm{K}_{\mathrm{\varepsilon}} \frac{\mathrm{U}^{3}}{2 L}\left[\frac{1}{\mathrm{C}_{\mathrm{d}}^{2}}-\mathrm{K}_{\mathrm{c}}-\left(1-\mathrm{s}^{2}\right)\right],
$$

with $K_{\varepsilon}=0.27$. The velocity $U$ is the liquid velocity at the injection nozzle, which has the length, $L$, and the diameter, $\mathrm{D}$. The discharge coefficient, the loss coefficient due to the nozzle entrance sharpness, and the downstream-to-upstream contraction area ratio of the injection nozzle are represented by $\mathrm{C}_{\mathrm{d}}, \mathrm{K}_{\mathrm{c}}$, and $\mathrm{s}$, respectively. Detailed derivations of these turbulence scales can be found in $[14,21]$.

The third term on the right hand side of equation (1) represents the contribution of the turbulence effect on the stripping rate of the parent drop. This turbulence term would accelerate the reduction in the parent drop radius with time and the modeled effect is consistent with the experimental observations [3, 18-20], in which the liquid jet has a short intact core length when the turbulence appears in the liquid jet due to the flow at a high Reynolds number or cavitation. It should be noted that for the non-turbulence case, equation (1) would become the original blob model of Reit, which only retains the effect of the surface wave motion on the primary breakup. Simiiar to the criterion set by Reitz, the parent drop would no longer strip its mass to create the product drop when the parent drop radius $a$ is less than the radius $r_{p}$ of the product droplet.

Studies of turbulent liquid jets $[5,6]$ indicated that the drops produced from the onset breakup regime have their sizes in the same order-of-magnitude of the turbulence length scale. On the other hand, Reitz set the radius of the product drop to be proportional to the wave length associated with the fastest growth rate. The above relations suggest that it is reasonable to formulate the radius of the product drops with the characteristic length scales for both the wave perturbation and turbulence phenomenon. For the present model, the reciprocal of the product drop radius is expressed by the sum of the reciprocals of the length scales associated with the surface wave instability and the turbulence motion with the inclusion of the respective weighting factors as follows

$$
\frac{1}{r_{p}}=\frac{c_{w}}{r_{w}}+\frac{c_{t}}{r_{t}} \Rightarrow r_{p}=\frac{r_{w} r_{t}}{\left(1-c_{t}\right) r_{t}+c_{t} r_{w}}
$$

Again, the radius of the product drop is $r_{p}$. The radius $r_{w}$ associated with the wave motion can be determined from the Reitz model. In the present phenomenological description, the kinetic energies associated to the surface wave and turbulence characteristics are used to weight both effects. The consideration of the kinetic energy level for this weighting is based on a reason of which the phenomenon of the larger kinetic energy motion would have a stronger influence in the liquid jet breakup process. Hence, the weighting coefficients $c_{w}$ and $c_{t}$ are determined by the kinetic energy ratio of the turbulence motion and wave perturbation. The value of $r_{1}$ is estimated from a probability density function (PDF), which was proportional to the ratio of the turbulence energy spectrum and the atomization time scale [14]. The notion for this representation was that an eddy motion with larger turbulent kinetic energy and a shorter atomization time most likely caused the drop breakup to occur more frequently than other ones containing the lower energy level and the longer atomization time. By assigning the wave number of the turbulence energy spectrum as the inverse of the product drop diameter, it can be shown in [21] that

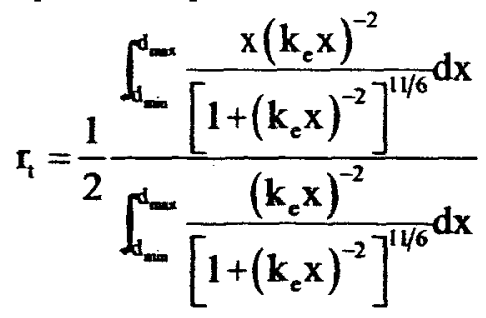

where $\mathrm{k}_{\mathrm{e}}=0.75 / \mathrm{L}_{\mathrm{t}}, \mathrm{d}_{\max }=$ maximum possible diameter of the product drop, and $\mathrm{d}_{\min }=$ smallest possible diameter of the product drop. The weighting coefficient $c_{1}$ is formulated with the kinetic energy terms related to the surface wave and flow turbulence levels as

$$
c_{t}=\frac{E_{k t}}{E_{k t}+E_{k w}}
$$

where 


$$
\begin{aligned}
& E_{k t}=\left(\frac{L_{t}}{\tau_{t}}\right)^{2} \\
& E_{k w}=\left(\frac{L_{w}}{\tau_{w}}\right)^{2}=\left(\frac{\Lambda^{2} \Omega}{a}\right)^{2} .
\end{aligned}
$$

Since the weighting coefficients represent degrees of the contributions of a particular physical phenomenon to the overall one, it is logical to assign a unity relation between them as $c_{w}+c_{t}=1$. With this relation, it is easy to recognize that for the constraint of $E_{k} \gg E_{k w}, r_{p}$ would become equal to $r_{t}$, and vice versa $r_{p}$ would approach $r_{w}$ if $E_{k t} \ll E_{k w}$. This ensures that the condition with a larger kinetic energy would have a more significant effect on the atomization process.

Along with the inclusion of the turbulence effect on the primary atomization process, the subject phenomenon also is considered in the secondary droplet breakup model, which is discussed in the next section. At any rate this new model requires the initial velocity fluctuation quantity of the product drops right after their formation. This quantity can be obtained by examining the energy conservation during the primary breakup process. Denoting $a_{b e}$ and $a_{a f}$ as the radii of the parent drop before and after its liquid stripping, respectively, the amount of the mass $\mathrm{m}_{\mathrm{br}}$ stripped from the parent drop is formulated as

$$
\mathrm{m}_{\mathrm{br}}=\frac{4}{3} \pi \rho_{1}\left(\mathrm{a}_{\mathrm{be}}^{3}-\mathrm{a}_{\mathrm{af}}^{3}\right)
$$

During this breakup process, the change in the individual energy forms of the parent drop is estimated as follows:

$$
\begin{array}{ll}
\text { Energy due to surface wave motion: } & \left(\Delta \mathrm{E}_{\mathrm{w}}\right)_{\mathrm{par}}=\mathrm{m}_{\mathrm{br}}(\Lambda \Omega)^{2} \\
\text { Surface tension energy: } & \left(\Delta \mathrm{E}_{\mathrm{surf}}\right)_{\mathrm{par}}=2 \pi \sigma\left(\mathrm{r}_{\mathrm{be}}^{2}-\mathrm{r}_{\mathrm{af}}^{2}\right) \\
\text { Turbulent kinetic energy: } & \left(\Delta \mathrm{E}_{\mathrm{tur}}\right)_{\mathrm{par}}=\mathrm{m}_{\mathrm{br}}\left(\frac{\mathrm{L}_{\mathrm{t}}}{\tau_{\mathrm{t}}}\right)^{2} \\
\text { Kinetic Energy of motion: } & \left(\Delta \mathrm{E}_{\mathrm{k}}\right)_{\mathrm{par}}=\mathrm{m}_{\mathrm{br}} \frac{\mathrm{V}^{2}}{2}
\end{array}
$$

For the product drop, the energy associated with the surface distortion is negligible as compared to the other energy forms, since the initial disturbance is small. Hence, the energy of the product drops is composed of the surface tension, turbulence, and kinetic motion as described below.

$$
\begin{array}{ll}
\text { Surface tension energy } & \left(E_{\text {surf }}\right)_{\text {prod }}=N_{\text {prod }} 2 \pi r_{p}^{2} \\
\text { Kinetic Energy of motion } & \left(E_{k}\right)_{\text {prod }}=m_{b r} \frac{V_{p r o d}^{2}}{2},
\end{array}
$$

where $V_{\text {prod }}$ and $N_{\text {prod }}$ are the velocity and number of product drops, respectively. They are evaluated as

$$
\begin{gathered}
V_{\text {prod }}=\sqrt{v^{2}+w^{2}} \\
N_{\text {prod }}=\frac{3 m_{b r}}{4 \pi \rho_{1} r_{p}^{3}}
\end{gathered}
$$

For the velocity, the product drops would carry the same velocity $\mathrm{V}$ of the parent along with two additional normal velocity components, $v$ and $w$, in reference to the trajectory of the parent drop. They are represented by

where

$$
\begin{aligned}
& \mathrm{v}=|\mathrm{V}| \tan (\theta / 2) \sin \phi \\
& \mathrm{w}=|\mathrm{V}| \tan (\theta / 2) \cos \phi,
\end{aligned}
$$




$$
\tan (\theta / 2)=A_{1} \Lambda \Omega / U
$$

A random parameter $\phi$ is chosen on the interval $(0,2 \pi)$. According to Reitz et al. [40], the constant $A_{1}$ depends on nozzle design. Its value is set equal to $A_{1}=0.188$ for the present study.

By equating the change in the energies of the parent drop with the energy of the product drops, the turbulent kinetic energy for the product drop is written as

$$
\left(E_{\text {tur }}\right)_{\text {prod }}=\frac{\left(\Delta \mathrm{E}_{\mathrm{w}}\right)_{\mathrm{par}}+\left(\Delta \mathrm{E}_{\mathrm{surf}}\right)_{\mathrm{par}}+\left(\Delta \mathrm{E}_{\mathrm{tur}}\right)_{\mathrm{par}}+\left(\Delta \mathrm{E}_{\mathrm{k}}\right)_{\mathrm{par}}}{N_{\text {prod }}\left(\left(\Delta \mathrm{E}_{\text {surf }}\right)_{\text {prod }}+\left(\Delta \mathrm{E}_{\mathrm{k}}\right)_{\text {prod }}\right)}
$$

This quantity is used to estimate the initial velocity fluctuation for the secondary breakup process, which is discussed next.

\section{B. T-TAB Model}

Studies $[7,8]$ of the turbulence effect on the liquid breakup suggest that the turbulence motion tends to weaken the surface tension force. In fact, it is well recognized that this surface tension keeps the liquid drop from being torn off, while the turbulence within the drop would promote the droplet disintegration process. To account for this behavior, a term $F_{b}$, representing a force associated with the effect of the turbulence on the droplet breakup, is introduced to the original TAB equation

$$
\mathbf{m} \ddot{\zeta}=\mathbf{F}+F_{\mathbf{t}}-\mathbf{k} \zeta-\mathrm{d} \dot{\zeta}
$$

It is assumed that the turbulence force $F_{t}$ is formed by a portion of the internal liquid turbulence energy. Another portion of subject energy decays through a dissipation process. This proposition is based on the experience that the turbulence motion behaves like a force participating in the droplet surface deformation and accelerating its surface displacement. Hence, it is proposed that the product of the turbulence force and the deformation rate of the droplet surface displacement must be related to the dissipation rate of the turbulence energy during this breakup process. This relationship is formulated as

$$
\mathrm{F}_{\mathrm{t}} \dot{\zeta}=\mathrm{C}_{\mathrm{t}} \mathrm{m} \varepsilon
$$

The dissipation rate of the turbulent kinetic energy per unit mass of individual drops is $\varepsilon$, and an empirical constant representing the proportionality of subject relationship is $\mathrm{C}_{\mathrm{t}}$.

It should be noted that without additional turbulence generation the internal turbulent kinetic energy would decay with time as the drops travel downstream. With an assumption of the turbulence within the drop being homogenous and isotropic, using the $\mathrm{k}-\varepsilon$ model [22], $\mathrm{k}$ can be expressed as a function of time:

$$
\begin{gathered}
k=\left[\frac{\varepsilon_{0}}{\left(k_{0}\right)^{C_{\varepsilon}}}\left(1-c_{\varepsilon}\right) t+\left(k_{0}\right)^{\left(1-C_{\varepsilon}\right)}\right]^{1 /\left(1-C_{\varepsilon}\right)} \\
\varepsilon=-\frac{d k}{d t}=-\frac{\varepsilon_{o}}{k_{0}^{C_{\varepsilon}}}\left[\frac{\varepsilon_{0}}{\left(k_{0}\right)^{C_{\varepsilon}}}\left(1-c_{\varepsilon}\right) t+\left(k_{0}\right)^{\left(1-C_{\varepsilon}\right)}\right]^{C_{\varepsilon} /\left(1-C_{\varepsilon}\right)}
\end{gathered}
$$

or

$$
\varepsilon=\varepsilon_{0}\left[\frac{k}{k_{0}}\right]^{c_{t}}
$$

The turbulence force, shown in equation (14), can be rearranged and written as

$$
\frac{\mathrm{F}_{\mathrm{t}}}{\mathrm{m}}=\mathrm{C}_{\mathrm{t}} \varepsilon_{\mathrm{o}}\left[\frac{\mathbf{k}}{\mathbf{k}_{\mathrm{o}}}\right]^{\mathrm{C}_{\mathrm{k}}} \dot{\zeta}^{-1}
$$


When the distortion displacement $\zeta$ is non-dimensionlized by $y=\zeta / C_{b} r_{p}$, equation (13) becomes

$$
\ddot{y}=\frac{C_{F}}{C_{b}} \frac{\rho_{g}}{\rho_{l}} \frac{W^{2}}{r_{p}^{2}}+C_{t} \frac{\varepsilon_{o}}{C_{b}^{2} r_{p}^{2}}\left[\frac{k}{k_{0}}\right]^{C_{\varepsilon}} \dot{y}^{-1}-C_{k} \frac{\sigma}{\rho_{l} r_{p}^{3}} y-C_{d} \frac{\mu_{l}}{\rho_{1} r_{p}^{2}} \dot{y}
$$

Except for the second term on the right hand side, equation (18) is the same as the governing equation of the TAB model. To estimate the post-breakup drop size, a similar method as used in the TAB model is employed for the calculation of Sauter mean radius.

$$
\mathrm{SMR}=\frac{4 \pi \mathrm{r}_{\mathrm{p}}^{3} \sigma}{\mathrm{E}_{\mathrm{pmr}}-\frac{\pi}{6} \mathrm{r}_{\mathrm{p}}^{5} \rho_{1} \dot{\mathrm{y}}^{2}}
$$

The total energy of the original drop prior to the breakup is $\mathrm{E}_{\mathrm{par}}$, and it is determined from equation the original TAB formulation

$$
E_{p a r}=E_{\text {surf }}+E_{\text {osc }}=4 \pi r_{p}^{2} \sigma+K \frac{\pi}{5} \rho_{1} r_{p}^{5}\left(\dot{y}^{2}+\omega^{2} y^{2}\right)
$$

The secondary breakup process is considered for all drops created from either the primary breakup or previous secondary droplet breakup and requires initial values of the turbulent kinetic energy and its dissipation rate. When a drop is created from the secondary atomization, it is assumed that the turbulence quantities of the parent drop are preserved and distributed evenly to its children drops. The turbulent kinetic energy $\mathbf{k}_{\mathrm{bt}}$ and corresponding dissipation rate $\varepsilon_{\mathrm{b}}$ of the parent drop at the breakup time are determined from equations (15) and (16). The time and initial turbulence values used in these equations are referenced to the time when the considered drop is formed. The formulations employed for this calculation are

$$
\begin{gathered}
k_{b t}=\left[\frac{\varepsilon_{0}}{\left(k_{0}\right)_{\varepsilon}^{C_{\varepsilon}}}\left(1-c_{\varepsilon}\right) t_{b t}+\left(k_{0}\right)^{\left(1-C_{\varepsilon}\right)}\right]^{1 /\left(1-C_{\varepsilon}\right)} \\
\varepsilon_{b t}=-\frac{\varepsilon_{o}}{k_{o}^{C_{c}}}\left[\frac{\varepsilon_{0}}{\left(k_{0}\right)^{C_{r}}}\left(1-c_{\varepsilon}\right) t_{b t}+\left(k_{0}\right)^{\left(1-C_{\varepsilon}\right)}\right]^{C_{\varepsilon} /\left(1-C_{\varepsilon}\right)}
\end{gathered}
$$

Again, $t_{b s}, k_{0}$, and $\varepsilon_{0}$ are the breakup time and the initial turbulence quantities of the parent drop. Based on the conservation of mass, the initial turbulent kinetic energy and dissipation rate of the new drop are simply formulated as

$$
k_{0}=\frac{r_{p}^{3}}{S_{M} R^{3}} k_{b t}, \quad \text { and } \varepsilon_{0}=\frac{r_{p}^{3}}{S M R^{3}} \varepsilon_{b t} .
$$

It should be emphasized that $k_{0}$ and $\varepsilon_{0}$ are denoted for the initial turbulence quantities of a corresponding drop type. The parameters in equations (20) and (21) belong to the parent drops, while they are for the product drops when used in equation (22).

When a drop of interest is directly produced from the primary atomization, its initial turbulence energy is determined from the energy conservation of the blob drop and its product drops at the breakup time. The formulation of the initial turbulent kinetic energy for the product drop, denoted as $\left(E_{t u r}\right)_{\text {prod }}$, has been derived and presented already in equation (12). Hence, the initial turbulent kinetic energy $\mathrm{k}_{\mathrm{o}}$ per unit mass for the drop is represented by

$$
k_{\mathrm{o}}=\frac{3}{4 \mu r_{p}^{3} \rho_{1}}\left(E_{\text {turb }}\right)_{\text {prod }} .
$$

The initial dissipation rate of the turbulence energy $\varepsilon_{0}$ is then determined from the relationship 


$$
L_{t}=c_{\mu} \frac{k_{o}^{3 / 2}}{\varepsilon_{o}} .
$$

With the assumption that $L_{t}=2 r_{p}, \varepsilon_{o}$ can be written as

$$
\varepsilon_{\mathrm{o}}=\mathrm{c}_{\mu} \frac{\mathbf{k}_{\mathrm{o}}^{3 / 2}}{2 \mathbf{r}_{\mathrm{p}}} .
$$

It should be emphasized that these two relationships are used for estimating the turbulence values of the new drops for the primary breakup only.

In an atomization process without the consideration of the droplet coalescence, the liquid drop breaks up into smaller drops. As a drop travels downstream its size reduces to the smallest possible dimension, and the turbulence activity within the drop also decays. Subsequently, it would lead to conditions in which the turbulence approaches its final energy cascade regime. Here, the molecular viscosity is effective in dissipating the turbulent kinetic energy, and the turbulence scales also reduce to the order of the Kolmogorov scale magnitude [23]. Hence, a criterion for eliminating the turbulence effect is postulated as follows

$$
\mathrm{SMD} \leq 4\left[\frac{\mu_{1}^{3}}{\rho_{1}^{3} \varepsilon_{\mathrm{o}}}\right]^{1 / 4}
$$

The bracketed term with its exponent represents the Kolmogorov length scale and is based on the liquid properties and the initial dissipation rate of the turbulence energy of the new drop immediately after the droplet breakup. When the above expression is satisfied, the turbulence effect is no longer valid and the T-TAB model would become the original TAB model. As seen from the results of all test cases in this study, the new drop size reaches the Kolmogorov scale in about two or three cycles of breakup steps.

\section{RESULTS and DISCUSSION}

\section{A. Primary Breakup Assessment (T-blob)}

Several experiments were selected to assess the current model. The flow conditions of the cases are summarized in Table 1. The turbulence scales, initial turbulent kinetic energy, and its dissipation rate required in T-blob model are estimated from equations (4) and (5) by knowing the injection nozzle configuration and its flow conditions. The values of the kinetic energy and the dissipation rate are listed in Table 1. It should be noted that for some test cases the measured data to calculate the initial turbulence values are not entirely reported. Subsequently, typical values are estimated and utilized in the calculation. Details of determining these initial values can be found in [21].

We first investigate the relative effects by comparing the mass stripping rate due to turbulence and surface wave as described in equation (1). The values of these terms for test cases $\mathrm{H}-1$ and $\mathrm{H}-3$ (see Table 1) are shown in Figure 1. We first investigate the relative effects by comparing the mass stripping rate due to turbulence and surface wave as described in equation (1). The values of these terms for test cases $\mathrm{H}-1$ and $\mathrm{H}-3$ (see Table 1) are shown in Figure 1. In this figure the values of these terms are plotted against the relative life time of the parent drop. This parameter is non-dimensionalized by the injection velocity and injection nozzle diameter. The results for the considered cases indicate that the surface wave perturbation has a considerable effect on the reduction rate of the parent drop size. The value of the wave motion term in the test case $\mathrm{H}-3$ is approximately two orders of magnitude greater than the one of the turbulence term. However, when the injection velocity increases from $86.4 \mathrm{~m} / \mathrm{sec}$ (case H-3) to 102.0 $\mathrm{m} / \mathrm{sec}$ (case H-1) the gap of these two values becomes smaller. This suggests that the value of the turbulence term rises at a faster rate than the one of the wave motion term, when the injection velocity increases. The reduction rate of the parent drop decreases with the increase of the velocity. Subsequently, the primary atomization process is elongated for the case of higher injection velocity. The reflection of the aforementioned effects can also be seen in Figure 2, where the parent drops predicted by the HK (blob) and T-blob models for test cases Hl through Y-3, are plotted. 
Table 1. Test cases and measured data used in the computations

\begin{tabular}{|c|c|c|c|c|c|c|c|c|}
\hline Case & H-1 & H-2 & $\mathrm{H}-3$ & $\mathrm{Y}-1$ & $\mathrm{Y}-2$ & $Y-3$ & $\mathbf{K}$ & $\mathbf{S}$ \\
\hline Nozzle Diameter (mm) & & 0.3 & & & 0.213 & & 0.24 & 0.15 \\
\hline Ambient Gas & & Nitrogen & & & arbon Dioxi & & Nitrogen & Nitrogen \\
\hline Ambient Pressure (MPa) & 1.1 & 3.0 & 5.0 & 4.5 & 2.5 & 0.5 & 2.17 & 1.5 \\
\hline Ambient Temperature (K) & & 298 & & & 298 & & 298 & 289 \\
\hline Density $\left(\mathrm{kg} / \mathrm{m}^{3}\right)$ & 12.36 & 33.70 & 56.17 & 72.61 & 40.34 & 8.07 & 24.51 & 16.84 \\
\hline Liquid Fuel & & Diesel Fuel & & & Diesel Fue & & Diesel Fuel & Diesel Fuel \\
\hline Density $\left(\mathrm{kg} / \mathrm{m}^{3}\right)$ & & 840 & & & 840 & & 840 & 840 \\
\hline Viscosity $\left(\mathrm{Kg} / \mathrm{m}^{-} \mathrm{s}\right)$ & & $2.9 \times 10^{-3}$ & & & $5.0 \times 10^{-3}$ & & $5.0 \times 10^{-3}$ & $2.9 \times 10^{-3}$ \\
\hline Surface Tension $(\mathrm{N} / \mathrm{m})$ & & $2.05 \times 10^{-2}$ & & & $2.06 \times 10^{-2}$ & & $2.06 \times 10^{-2}$ & $2.05 \times 10^{-2}$ \\
\hline Injection Velocity $(\mathrm{m} / \mathrm{s})$ & 102.0 & 90.3 & 86.41 & & 185.42 & & 133.81 & 183.00 \\
\hline Initial Turbulence Quantity & & & & & & & & \\
\hline Kinetic Energy $\left(\mathrm{m}^{2} / \mathrm{s}^{2}\right)$ & $2.88 \times 10^{2}$ & $2.26 \times 10^{2}$ & $3.94 \times 10^{1}$ & $1.58 \times 10^{3}$ & $1.58 \times 10^{3}$ & $1.58 \times 10^{3}$ & $5.22 \times 10^{2}$ & $4.64 \times 10^{2}$ \\
\hline $\begin{array}{l}\text { Dissipation Rate of Kinetic } \\
\text { Energy }\left(\mathrm{m}^{2} / \mathrm{s}^{3}\right)\end{array}$ & $1.06 \times 10^{8}$ & $7.34 \times 10^{7}$ & $1.22 \times 10^{7}$ & $1.49 \times 10^{9}$ & $1.49 \times 10^{9}$ & $1.49 \times 10^{9}$ & $3.14 \times 10^{8}$ & $6.11 \times 10^{8}$ \\
\hline Reference & \multicolumn{3}{|c|}{ Hiroyasu et al. [24] } & \multicolumn{3}{|c|}{ Yule et al. [25] } & Koo [30] & $\begin{array}{l}\text { Schneider } \\
\text { [31] }\end{array}$ \\
\hline
\end{tabular}

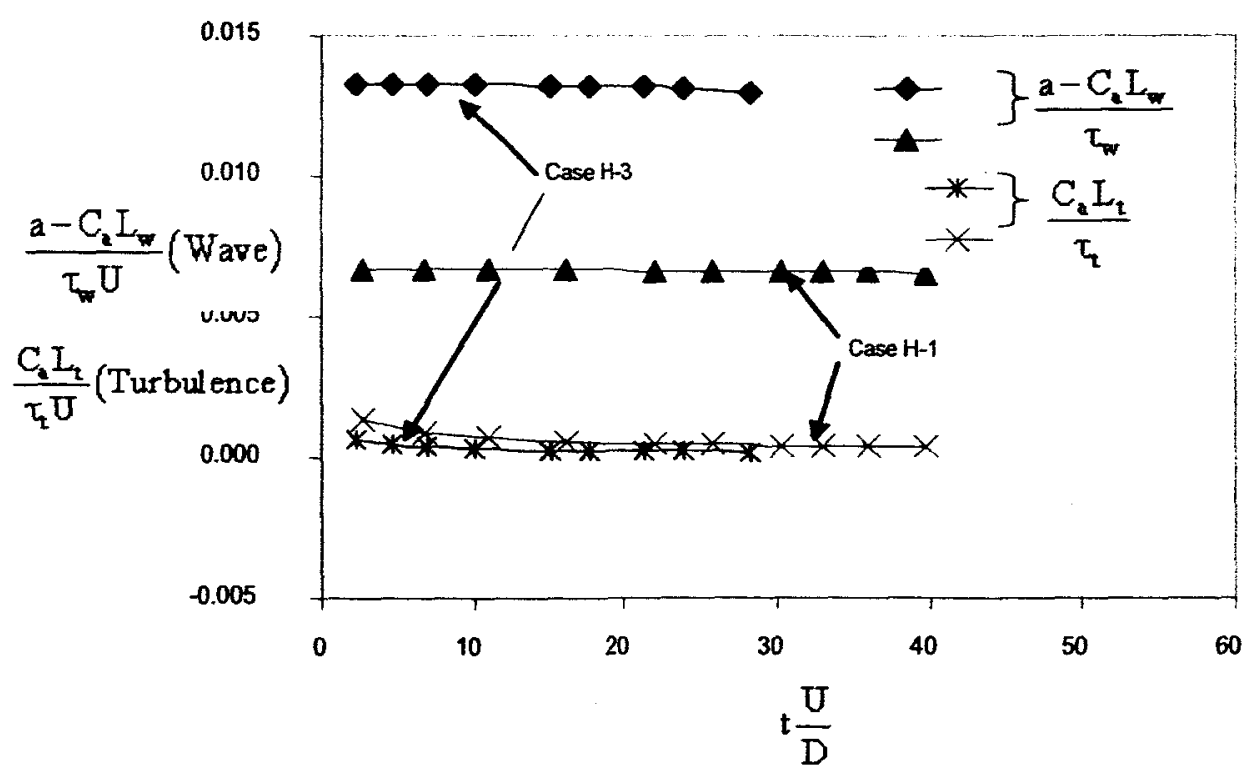

Figure 1. Values of the wave motion and turbulence terms on the reduction rate

11

American Institute of Aeronautics and Astronautics 
It is evident from Figure 1 that the reduction rate in the parent drop size is nearly constant with time. This trend is due to the fact that the term, associated with the surface wave motion, is determined from the wave length and its fast growth rate, which are not a function of time. On the other hand, the turbulence term is time dependent. Combining these two equations when calculating the tubulence term, however, only offers a slight decrease in its value with time. In addition, the end of the parent drop breakup occurs in a relatively short time. Hence, the results show the rate of change in the parent drop size is almost constant. Therefore, the curves of the parent drop diameters as shown in Figure 2 are nearly straight downward. In all test cases, the T-blob model predicts the completion of the parent drop breakup slightly earlier than the prediction from the blob model.

It should be pointed out that the T-blob model only describes the primary breakup. In the actual measurement, it is, however, difficult to separate other physical phenomena, such as the secondary droplet breakup, etc. Therefore, only the intact core length of the liquid jet can be legitimately used to compare the measurement and prediction. So, an available correlation [26] of the intact length, which has been widely used in literature, is used to compare with the present prediction. The correlation is shown below

$$
L_{\text {jet }}=C_{j e t} \sqrt{\rho_{1} / \rho_{g}} D
$$

The intact length is denoted by $L_{j e t}$ and the constant $C_{j e t}$ has a value of 10.0. Due to the nature of the implementation of the $b l o b$ and T-blob models, it is reasonable to define the intact length of the liquid jet as the traveling distance of the injected $b l o b$ droplets during this process. The non-dimensional intact length $L_{j e t} / D$, predicted by both models, plotted against the square root of the density ratio $\sqrt{\rho_{1} / \rho_{g}}$, are shown in Figure 3 .
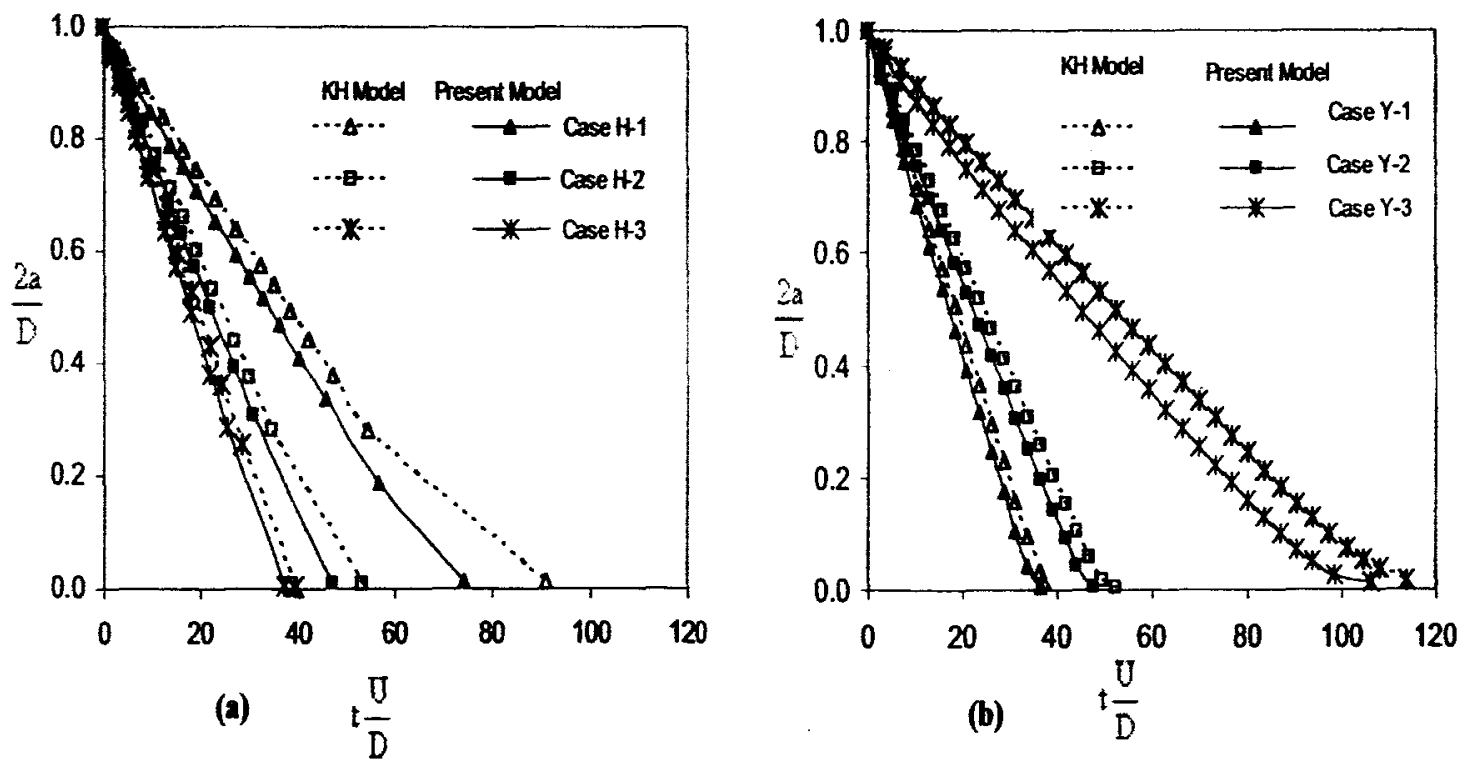

Figure 2. Rate of change in parent drop size predicted by $\mathrm{KH}$ (blob) and T-blob model

(a) Test cases $\mathrm{H}-1, \mathrm{H}-2, \mathrm{H}-3$. (b) Test cases $\mathrm{Y}-1, \mathrm{Y}-2, \mathrm{Y}-3$ 


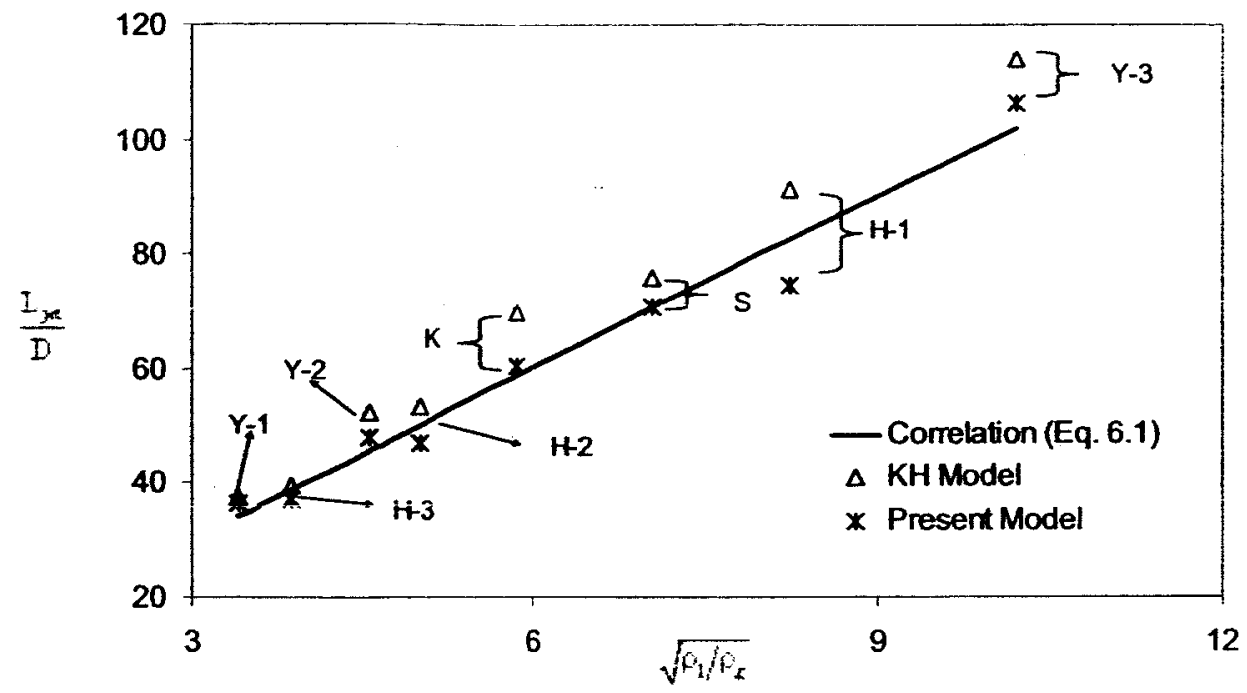

Figure 3. Comparison of predicted and correlation intact lengths of the liquid jet

The predicted results from the blob and T-blob models reasonably agree with the correlation curve. In general, the data points from the T-blob model are closer to the correlation line in comparison with the ones from the blob model. Furthermore, the T-blob model predicts a shorter intact length than the blob model does. This prediction is consistent with the measured data trends observed by other authors $[3,26]$.
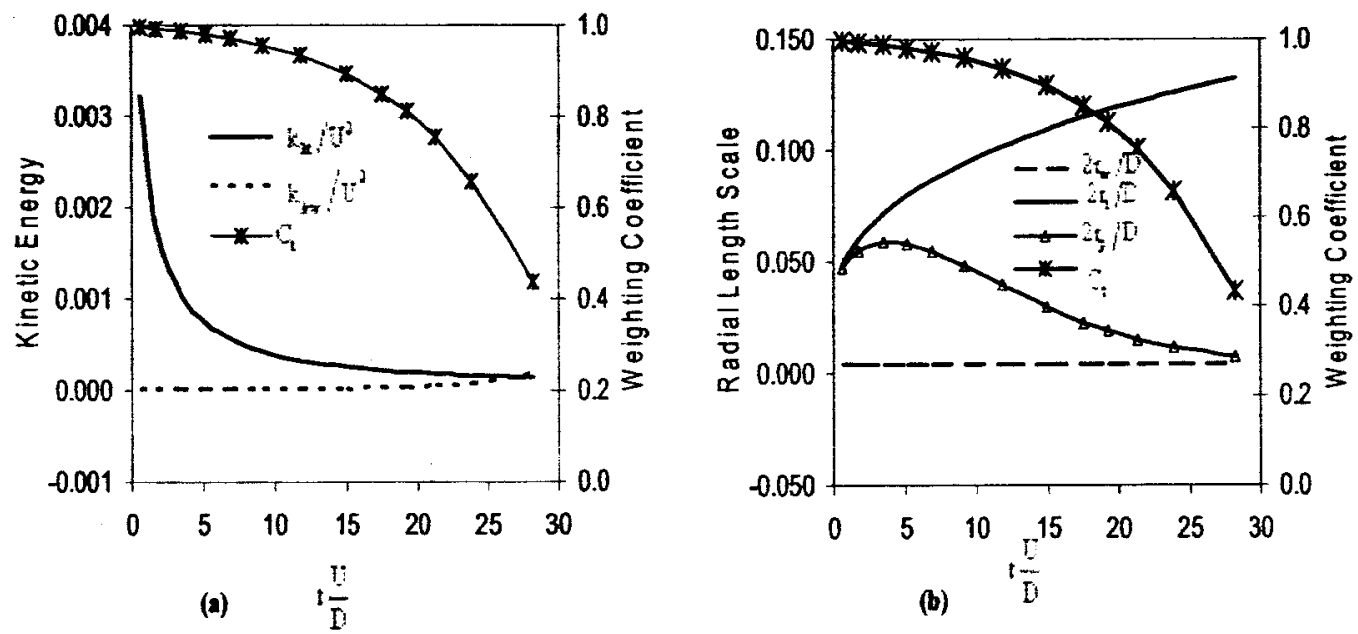

Figure 4. Parameters used to determine product drop size

(a) Kinetic energies and weighting coefficient;

(b)Radial length scales and weighting coefficient

In the present model, both the liquid jet surface wave perturbation and the turbulence motion play a part in forming the product drops. Their drop size, as shown in equation (6), is composed of the two radial length scales $r_{w}$ and $r_{t}$. They are weighted by the kinetic energies of the respective phenomena when used to determine the radius of the product drops. The values of subject parameters for the test case H-3 are plotted in Figure 4. The curve showing the kinetic energy of the surface wave motion in Figure 4(a) is every much constant with time, because the surface wave length and its corresponding growth rate are not a function of time. On the other hand, the kinetic energy associated with the turbulence is initially at a high level due to the turbulence developed at the injector nozzle exit. 
However, its value rapidly decreases through the dissipation process and approaches the level of the surface wave motion. This trend has led to define the weighting coefficient curve (see equation (8)), which starts at the value near unity and then quickly reduces its value to one half at the end of the product drop generation. Figure 4 (b) presents the radial length scales formulated from the two considered motions. Using the same previous argument of the surface wave length being constant, it is easy to recognize that the radial length scale $r_{\mathrm{w}}$ associated with this motion is more or less constant with time. In contrast, the value of $r_{l}$ involving the turbulence increases with time since the turbulence length scale $L_{1}$ increases with time and the radial length $r_{1}$ is a function of $L_{1}$. When applying the weighting coefficient $C_{1}$ (its curve is also shown in Figure 4 (b)) in the determination of the product drop size, the resultant value of $r_{p}$ initially rises and then gradually drops due to the reduction of $C_{r}$. This trend can be interpreted such that the high turbulence intensity at the initial primary breakup stage controls the formation of the product drop size. As the parent drop travels downstream the turbulence dissipates. Then, this drop formation process is gradually dominated by the surface wave perturbation. For the test case $\mathrm{H}-3, \mathrm{r}_{\mathrm{w}}$ determined from the Reitz model has a value less than $1 \mu \mathrm{m}$ while $r_{t}$ formulated by Huh et al . [14] is approximately $20 \mu \mathrm{m}$. Due to the ignorance of the drop collision and coalescence effects, the value of $r_{t}$ is more representative of the drop size found in the measured data.

This same value is also predicted by the T-blob model at the initial product drop formation. However, the drop size continues to decrease to the level predicted by the blob model. As the parent drop strips its mass and consequently reduces its size when traveling downstream without coalescence, it is logical to expect that the product drop size should decrease also in this process. It should be noted that the same trends of all parameters describing test case $\mathrm{H}-1$ are also observed in all the test cases shown in Table 1. The prediction of the product drop sizes for test cases H-1 to Y-3 is displayed in Figure 5.

The results indicate that when liquid is injected at a higher velocity (test cases $\mathrm{H}-1$ and $\mathrm{Y}-1$ ) product drops are initially formed with a larger size and this product drop formation process is taken for a longer time in comparison to the process at the lower injection velocity (test cases $\mathrm{H}-3$ and $\mathrm{Y}-3$ ). Similar to the observation from Figure 4, the results shown in Figure 5 reveal that the product drop size predicted from the T-blob model is approximately one order magnitude larger than the one from the blob model. Again, by ignoring other effects, the drop size estimated from T-blob is consistent with the experimental data.
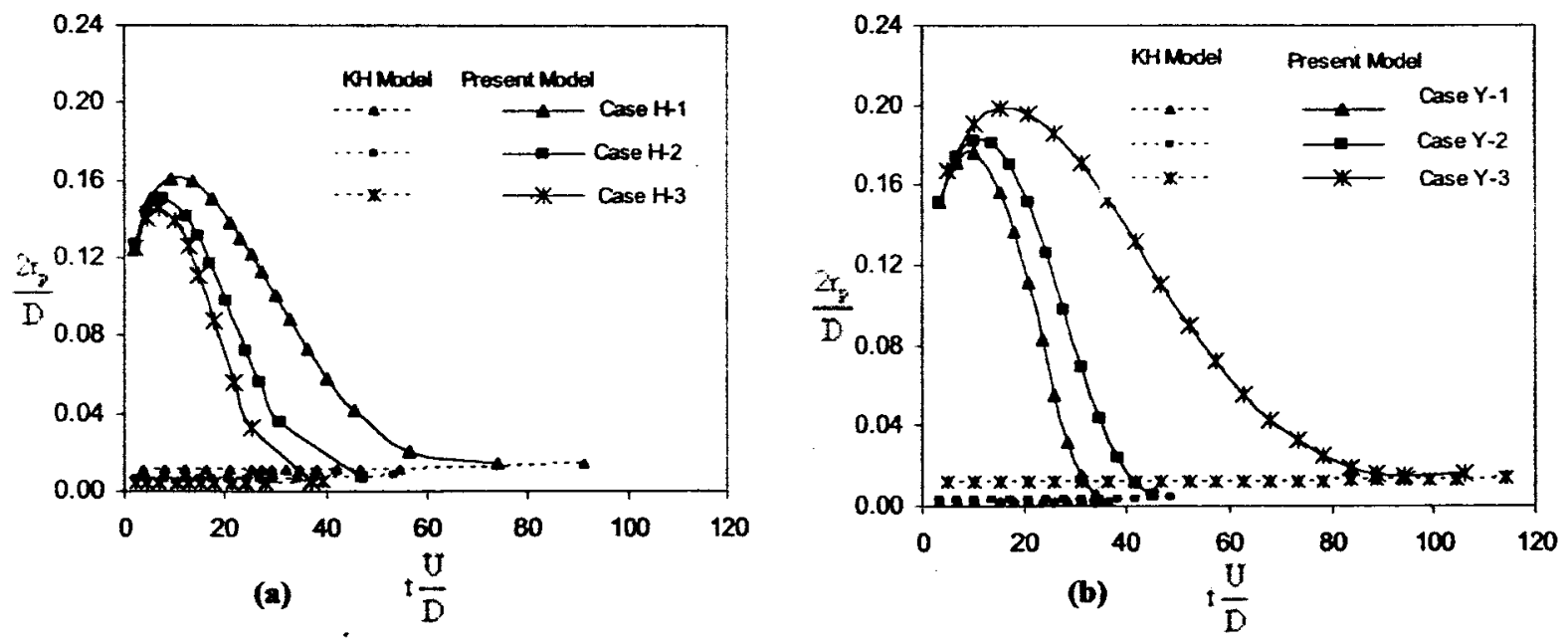

Figure 5. Product drop size predicted by $\mathrm{KH}$ and T-blob models

(a) Cases H-1, H-2, H-3, (b) Cases Y-1, Y-2,Y-3

As the product drops from the primary liquid jet breakup continue traveling downstream these drops are exposed to external as well as internal forces. Subsequently, they may undergo additional breakup cycles. This phenomenon is driven by the secondary breakup mechanism. The modeling assessment of which will be discussed in the following section. 


\section{B. Secondary Droplet Breakup Model (T-TAB)}

To assess the secondary droplet breakup model, the breaking up of an isolated liquid droplet traveling in a gaseous medium was numerically calculated [21]. The model is computed with a temporal variation of relative velocity between the drop and gas medium. Hence, the force terms in the governing equation of the drop breakup process can be predicted in a more realistic manner.

We first evaluate the turbulence force constant described in equation (14). The coefficient $C_{1}$ represents the proportionality between the dissipation rate of the turbulent kinetic energy and the turbulence energy participating in the droplet deformation. For the present study, the value of $C_{t}$ is estimated from measurements of drop breakup provided by Chou et al. [27]. In their shock tube experiment, the breakup of a single liquid drop in a flow behind a moving shock wave was investigated. The gas in the driven section flows at a velocity of approximately $80.8 \mathrm{~m} / \mathrm{s}$ with a density of $1.48 \mathrm{~kg} / \mathrm{m}^{3}$. The average product drop SMR of the entire secondary breakup process and the parent drop axial velocity $u_{p}$ are collected and reported in forms of the following correlations

$$
\begin{gathered}
\frac{S M R}{r_{p}}=2.6 \frac{t_{b l}}{t^{*}}\left[\frac{\rho_{1}}{\rho_{g}}\right]^{1 / 4} \sqrt{\frac{\mu_{1}}{2 \rho_{1} r_{p} W_{o}}} \\
u_{p}=3.75 \frac{t}{t^{*}} \sqrt{\frac{\rho_{1}}{\rho_{g}}}
\end{gathered}
$$

The characteristic breakup time $t^{*}$ is defined as $2 r_{p} \sqrt{\rho_{1} / \rho_{g}} / W_{o}$. The time $t$ is referenced to the time at which the drop is introduced to the flow field, while $t_{b t}$ is the time when the secondary breakup is complete. The results from subject experiment suggested that $t_{\mathrm{b}} / \mathrm{t}^{*}$ was approximately equal to 5.5 . Therefore, this value is used in equation (25) for estimating the SMR of the product drops. In the present study, the relative velocity of the parent drop is computed from equation (26) for the secondary breakup models (TAB and T-TAB). Due to the lack of measured droplet turbulence quantities, the initial turbulent kinetic energy $k_{0}$ used in the T-TAB equation is estimated from the parent drop velocity. Based on numerical simulations of the primary atomization for cases listed in Table 1, the ratio of the fluctuation velocity within the drop and the drop velocity ranges from 0.09 to 0.11 [21]. The ratio of 0.1 is selected for determining the initial droplet turbulence energy. Since Chou et al. [27] and Liang et al. [28] indicated that the drop starts the breakup at $t / t^{*}=1.5$, the initial drop velocity is predicted for this particular time. The initial dissipation rate $\varepsilon_{0}$ required in T-TAB equation was also estimated based on the discussion in Section II-B. A single water drop of $590 \mu \mathrm{m}$ in diameter has been analyzed using the density, viscosity, and surface tension constants of $997 \mathrm{~kg} / \mathrm{m}^{3}, 8.94 \times 10^{-4} \mathrm{~kg} / \mathrm{ms}$, and $70.8 \times 10^{-3} \mathrm{~N} / \mathrm{m}$, respectively. The product drop size for this case at various $C_{7}$ values is plotted in Figure 6. Since the correlation of the experimental data is not related to $C_{7}$ at all, its value shown in this figure at $S M R / r_{p}=0.135$ remains constant. Note that the TAB model does not carry the turbulence term; therefore, the results from this model would also not vary with $C_{t}$. Furthermore, TAB predicts the product drop size at $S M R / r_{p}=0.145$, which is roughly $7.5 \%$ larger than the measured value. The solid-line curve in Figure 6 displays the results of the present model with a variation of $C_{1}$. As would be expected, the T-TAB model reproduces the same results of the TAB model when $C_{t}=0$. With an increase in the value of $C_{t}, T-T A B$ predicts a smaller product drop size. In other words, the results of the present model suggest that the secondary atomization process would create a smaller drop size when stronger turbulence exists within the parent drop. From the plot, it is evident that the predicted SMR of the product drop matches the measured data for $C_{1}=0.19$. Consequently, this value is selected for the turbulence constant used in this study. 


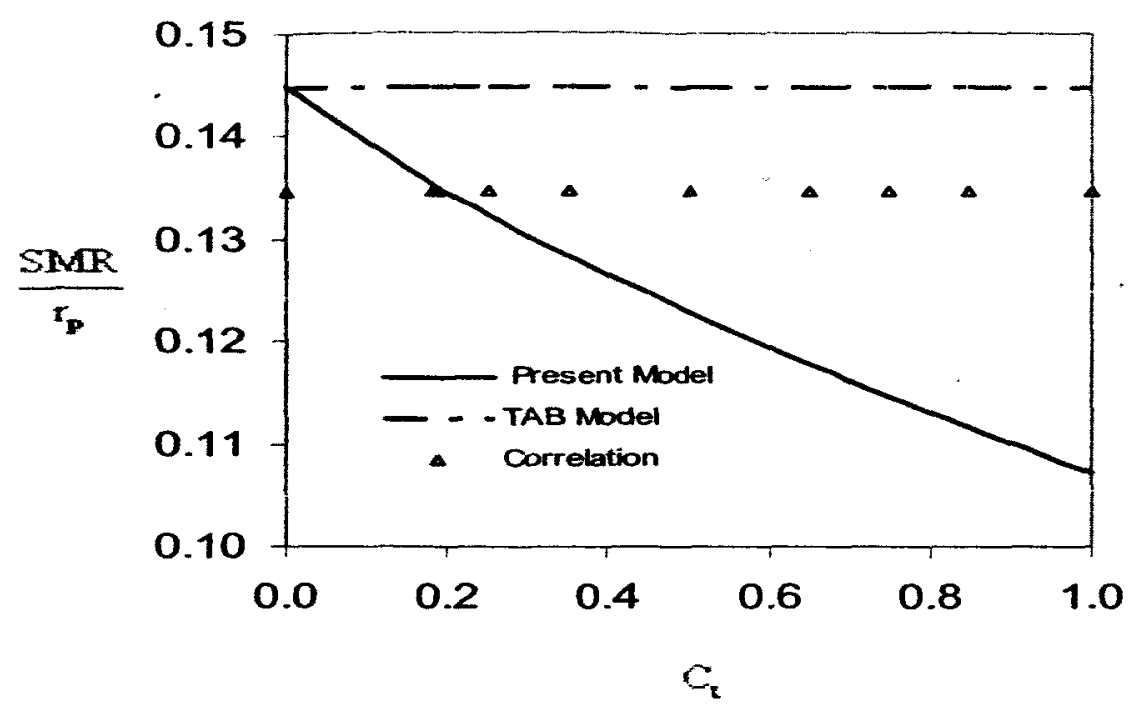

Figure 6. Variation of product drop size due to turbulence force coefficient $C_{t}$

To further assess the T-TAB model, the relative velocity between the drop and the gaseous environment is calculated from following relationship:

$$
\frac{\mathrm{dW}}{\mathrm{dt}}=-\frac{3 \mathrm{C}_{\mathrm{D}} \rho_{\mathrm{g}}}{8 \mathrm{r}_{\mathrm{p}} \rho_{1}} \mathrm{~W}^{2}
$$

The drag coefficient $C_{D}$ is determined from a model offered by Liu $e t$ al. [29]. In this model, the drag of a deformed droplet is composed of two parts. The first part is the drag of a rigid sphere, while the second part involves drag associated with the drop surface deformation. The relationship is represented as

$$
C_{D}= \begin{cases}\frac{24}{\operatorname{Re}}\left(1+\frac{1}{6} \operatorname{Re}^{2 / 3}\right)(1+2.632 y) & \operatorname{Re}<1000 \\ 0.424(1+2.632 y) & \operatorname{Re}>1000\end{cases}
$$

The Reynolds number $\mathrm{Re}$ is defined as $2 \rho_{\mathrm{g}} \mathrm{Wr}_{\mathrm{p}} / \mu_{\mathrm{g}}$. The droplet distortion displacement $\mathrm{y}$ is calculated from equation (18). When the value of $C_{D}$ is computed from a previous time step, equation (27) becomes an ordinary differential equation and can be solved easily [21]. Since the initial turbulence quantities are required for the T-TAB model it is worthwhile to conduct a sensitivity study of the turbulent kinetic energy $k_{o}$ on the predicted results. Variations of the product drop size and drop breakup time at different $k_{o} / .5 \mathrm{~mW}_{0}^{2}$ values for test case $\mathrm{H}-3$ in Table 1 are plotted in Figure 7.

The $x$-coordinate represents the ratio of the initial turbulence energy $k_{0}$ and droplet motion kinetic energy, $1 / 2 \mathrm{~mW}_{0}^{2}$. The curves of both product drop size and breakup time show that these quantities are decreasing almost linearly with an increase of the initial turbulence energy, except for $k_{0} \sim 0$. These results suggest that the level of the initial turbulence within the liquid has a strong effect on the droplet breakup. It is also of interest to note that the secondary atomization process generates smaller drops with the inclusion of turbulence. Also, it takes a shorter time to break up the parent drop when turbulence is considered. When implementing the secondary atomization model with the CFD code, the initial turbulence quantities are estimated from the process of the parent drop formation. As discussed in [21], CFD results showed $\mathrm{k}_{\mathrm{o}} / .5 \mathrm{~mW}_{\mathrm{o}}^{2}$ ranges from 0.09 to 0.11 for test case $\mathrm{H}-3$ when the drop is formed during the primary atomization. Hence, it is reasonable to assign $\mathrm{k}_{\mathrm{o}} / .5 \mathrm{~mW}_{\mathrm{o}}^{2}=0.1$ for the current study here. 
In the T-TAB model, the droplet distortion motion is driven by the interaction of aerodynamic, turbulence, surface tension, and viscosity events. The budget of these quantities with time is shown in Figure 8.

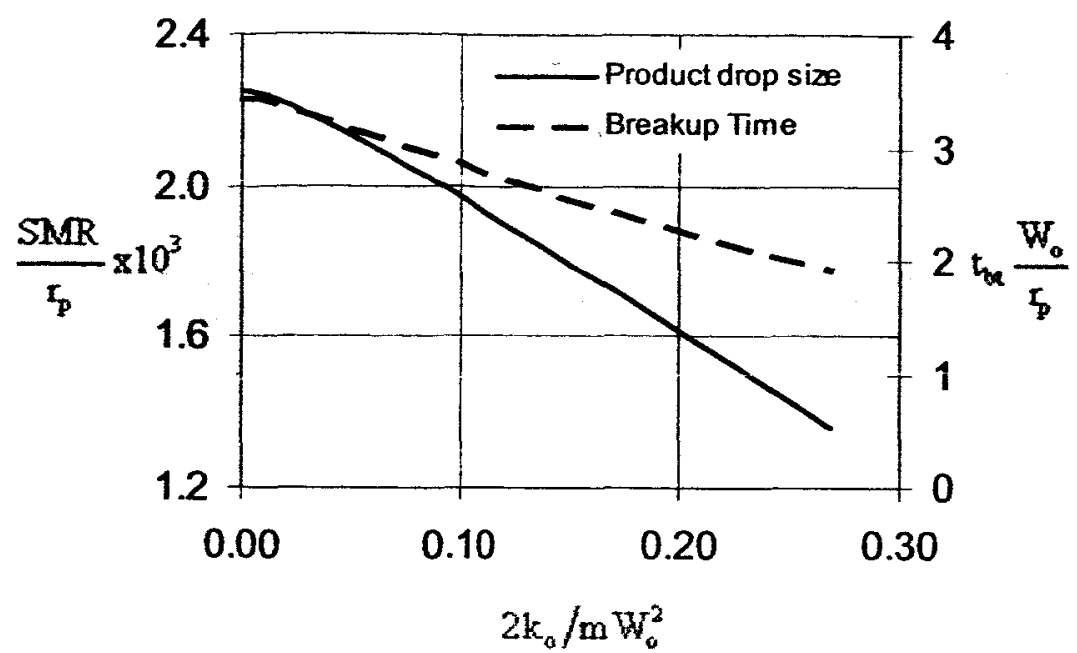

Figure 7. Product drop size and breakup time of various initial turbulent kinetic energy

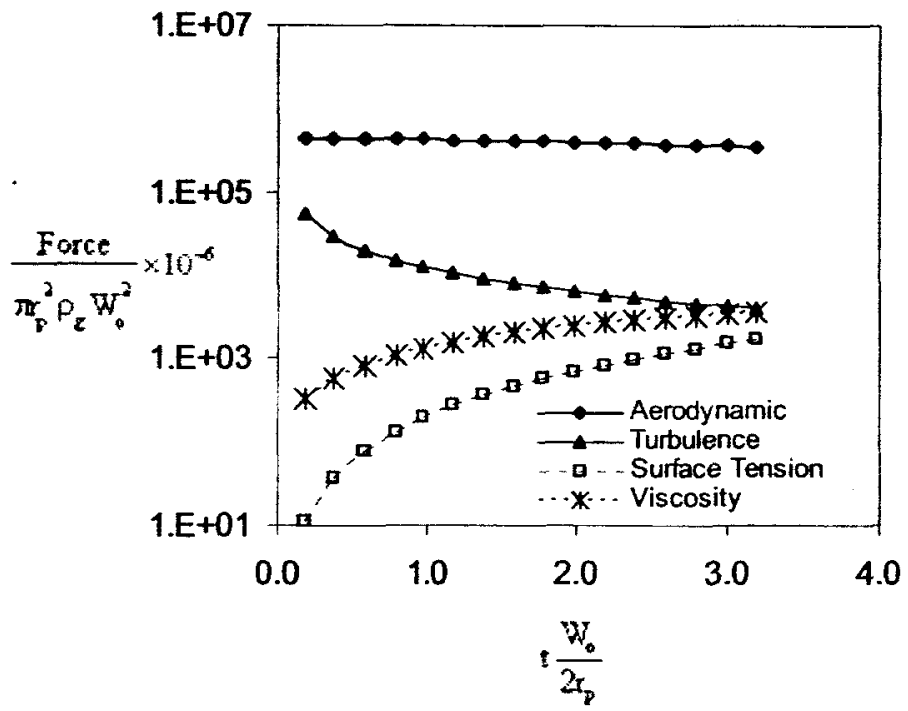

Figure 8. Forces generating droplet distortion based on T-TAB

This plot depicts that the aerodynamic force has a strong effect on the droplet breakup process. The flow conditions for the test case $\mathrm{H}-3$ are at a Reynolds number $\left(2 \rho_{\mathrm{g}} \mathrm{r}_{\mathrm{p}} \mathrm{W}_{\mathrm{o}} / \mu_{\mathrm{g}}\right)$ and Weber number $\left(2 \rho_{\mathrm{g}} \mathrm{r}_{\mathrm{p}} \mathrm{W}_{\mathrm{o}}^{2} / \sigma\right)$ of $3.8 \times 10^{3}$ and 80.7 , respectively. It should be noted that the time derivative of the distortion displacement $y$, which can be seen later in Figure 9, starts at a small value and increases with time. Since the turbulence force is inversely proportional to the $y$-derivative, this force consequently is very high at the initial time and rapidly changes to a smaller value. In contrast, the surface tension and viscosity forces are initially small and change to large values with time, because they are directly proportional to $y$ and $\dot{y}$, respectively. For the case without any turbulence, the drop distortion motion, according to the breakup model, is created by the difference of forces between the aerodynamics 
and the sum of the surface tension and viscosity. Obviously, this process is accelerated when turbulence is considered.

A comparison of $y$ and $\dot{y}$ computed from TAB and T-TAB are presented in the Figure 9. When these forces act on the drop, the distortion displacement $y$ would increase with time. Moreover, the imbalance of these forces would lead to an increase in the time derivative of this displacement $\dot{y}$. When y reaches the value of one, the parent drop produces into smaller drops. Figure 9 also reflects a consistency with the results observed in Figure 8 . Because of an additional turbulence term considered in the T-TAB model, it predicts higher values of $y$ and $\dot{y}$ at a given time in comparison to the results of TAB. Subsequently, the breakup time determined from the T-TAB model is shorter than the one from the TAB model.

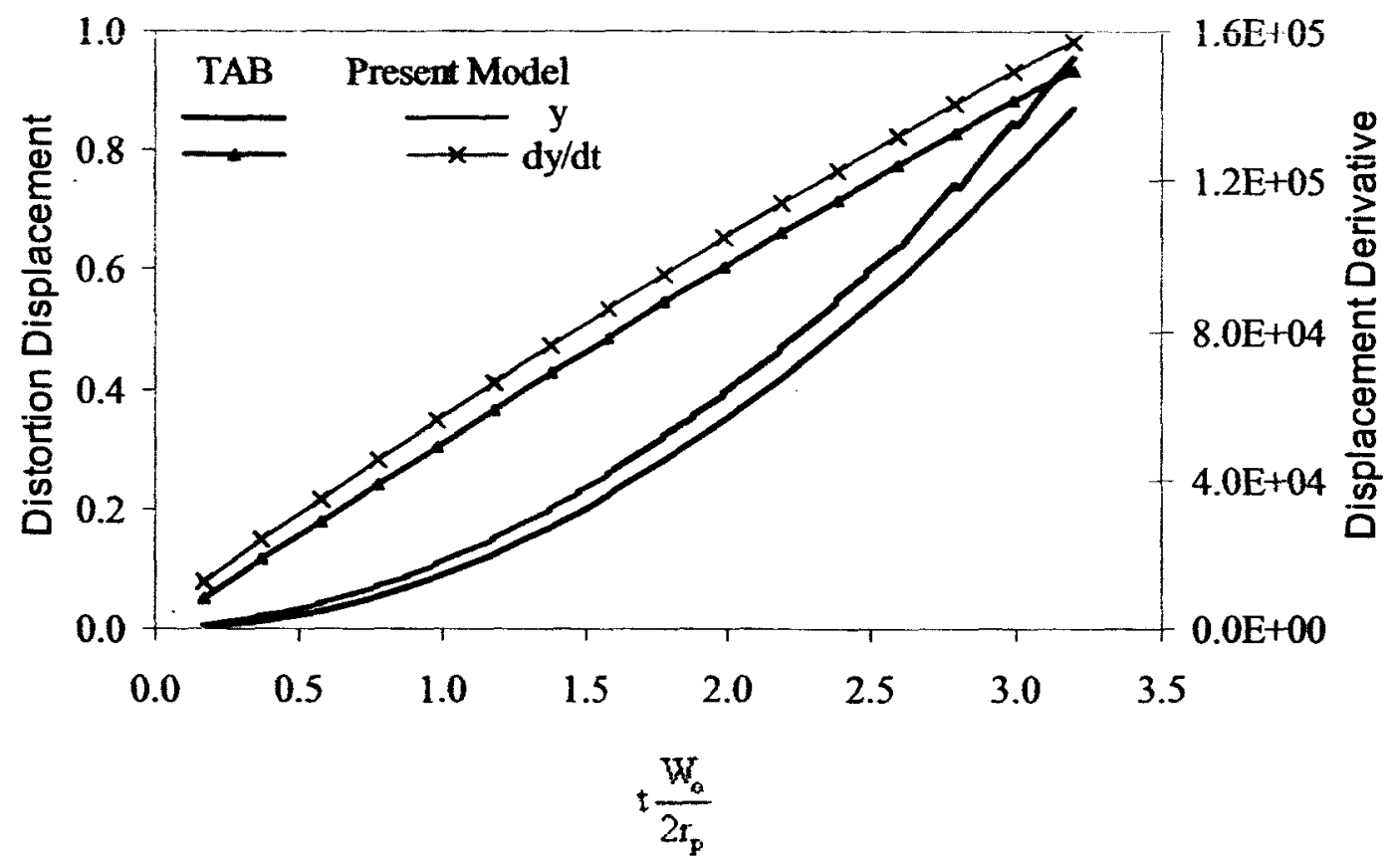

Figure 9. Comparison of the droplet distortion displacernent $(y)$ and its derivative $(\dot{y})$ predicted by $T A B$ and $T-T A B$

Although no measured data of the secondary breakup are available for the test cases listed in Table 1, they are still computed with both the TAB and T-TAB models to assess the variation in their results. Figure 10 shows a comparison of the predictions from TAB and T-TAB. The $x$-coordinate of the plot shows the ratio of aerodynamic and turbulence forces. This parameter is selected for plotting since the aerodynamic force is generally dominant in the droplet breakup process. The right and left vertical coordinates present the percentage differences in the product drop size and breakup time, respectively, predicted from TAB and the present model. The negative scales of these two coordinates indicate that the present model predicts smaller values. The results also suggest that the large variation of the two predictions appears at the low aerodynamic/turbulence force ratio where the turbulence action becomes significant in comparison to the aerodynamic force. However, the variation becomes smaller with an increase of this ratio value where the aerodynamic force is dominant. This trend suggests that the large variation between the two predictions results from the large turbulence force. When the aerodynamic force is significantly higher then it plays a key role in the droplet breakup. For this condition, the results from TAB and T-TAB are not much different regardless of how high the value of the turbulence force is. 


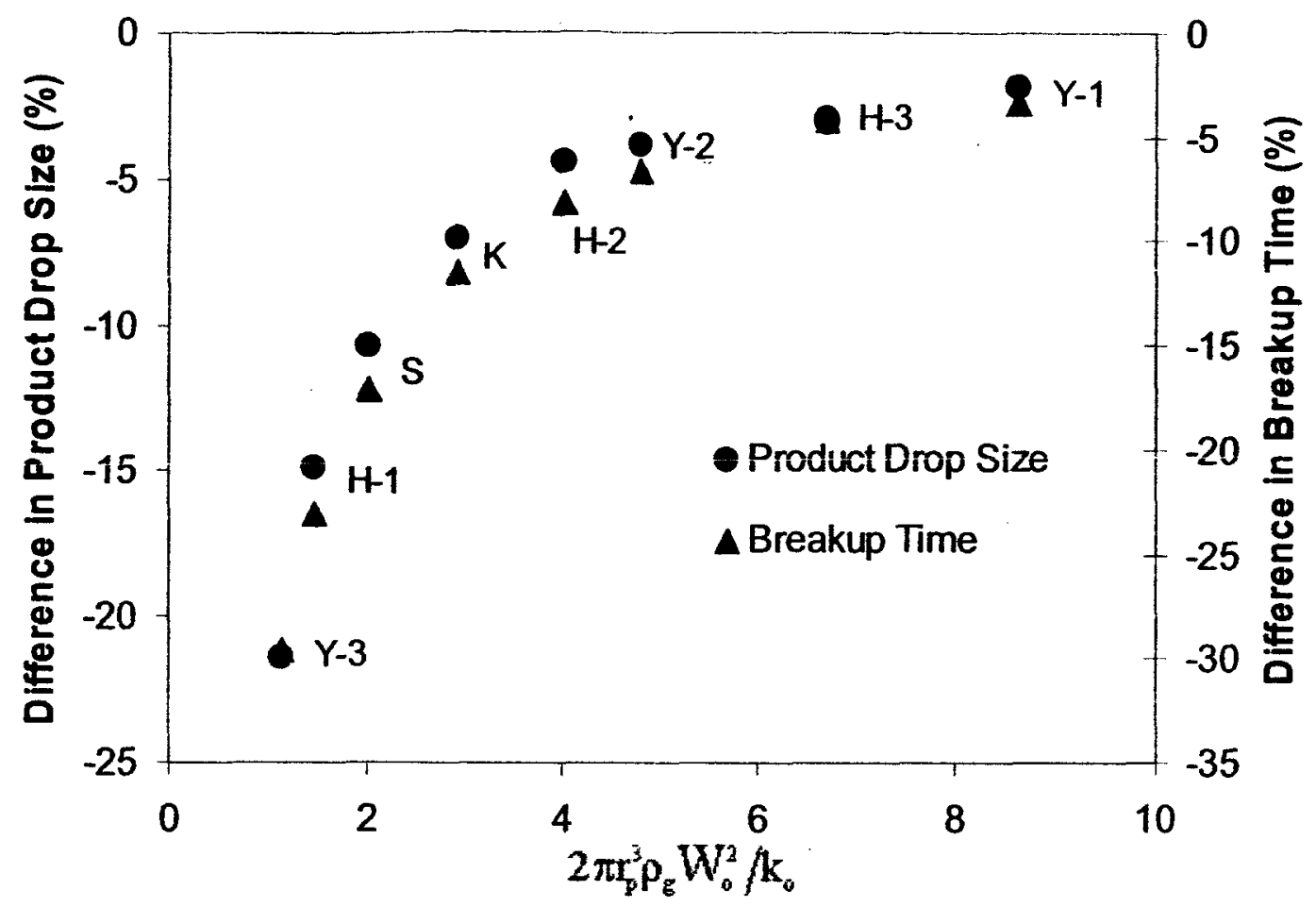

Figure 10. Difference in product drop side and breakup time predicted by TAB and T-TAB

\section{CONCLUSIONS}

We have presented two new models for modeling turbulence effect in atomizing liquid spray. In the $T$-blob model for primary breakup, both the characteristic length and time scales of the surface wave perturbation and the ones of the turbulence motion are combined in such a way that their contribution to the breakup mechanism is weighted by means of the kinetic energy. It has been observed that the initial turbulence quantities play a key role on the jet disintegration. Their values are estimated from the geometry and flow conditions of the injection nozzle. For the secondary droplet breakup event, an additional force term, composed of the deformation rate of the surface distortion, the dissipation rate of the turbulent kinetic energy, is incorporated into the T-TAB model. Assessments of these two proposed atomization models have been performed and several observations and conclusions can be drawn as follows:

1. In the primary atomization regime, the predictions generally show that the intact core length of the turbulent liquid jet is slightly shorter than without the turbulence consideration. In fact, at least in the test cases considered in this study, the combined characteristic scales used in the T-blob model result in a shorter jet breakup time, leading to a shorter intact core as compared to the blob model.

2. When the turbulent liquid jet disintegrates and then forms droplets, these drops are generally larger than the drops produced by a non-turbulent jet. The computational results of the present models reflect this finding, particularly in test case $\mathrm{H}-3$, where the product drop size predicted from the T-blob model is as much as one order of magnitude larger than the size predicted from the blob model.

3. In general, the turbulence inside the liquid jet affects the primary breakup of the liquid jet. This turbulence is characterized by its fluid properties, gas flow conditions, and initial turbulence quantities, $k_{0}$ and $\varepsilon_{o}$. In turn, these initial quantities are dependent on the flow conditions and the geometry of the injection nozzle.

4. According to the results from the T-TAB model, the turbulence inside the parent drop also plays a role in the droplet surface distortion. Similar to the primary breakup, the level of the turbulence effect on the secondary droplet breakup process depends much on its initial turbulence values. However, this turbulence

19

American Institute of Aeronautics and Astronautics 
effect is diminished after a few droplet breakup cycles [21]. This is due to the turbulence reduction from one generation of drops to the next, which is purely based on the current assumptions of estimating the initial turbulence quantities.

5. Close examinations of the predicted results for several test cases suggest that the values of the forces participating in the droplet deformation vary significantly at the initial time. The aerodynamic and turbulence forces are the strongest among them. However, the turbulence force approaches a same order of magnitude as the surface tension and viscous forces at the breakup time, while the magnitude of the aerodynamic force is still maintained. This observation reconfirms that the turbulence has a considerable influence on the secondary breakup, when compared with the surface tension and viscosity effects. However, the aerodynamic force also still plays a dominant role in the breakup process.

6. In contrast to the characteristics of liquid jet disintegration, the secondary droplet breakup mechanism produces small drops with a short breakup time when turbulence is considered. The turbulence force term in the present model is constructed to promote the droplet surface distortion. That formulation leads to predict a smaller drop size and shorter breakup time than the results obtained from the existing TAB model.

This research provides a basic framework to enhance the atomization models and the two new models presented in this paper have been incorporated into CFD code for full-field atomization simulation. These results will form the basis of a future communication.

\section{Acknowledgments}

This research is supported by NASA Marshall Space Flight Center in Huntsville, Alabama under a full time study program. Technical consultations of Dr. D.S. Crocker, Dr. B. Zuo, and Dr. S. Kim of CFD Research Corporations in this study are acknowledged. Finally, helpful assistance of librarians at Redstone Scientific Information Center in Huntsville, Alabama is appreciated.

\section{References}

1. Chen, T.F., Davis, J.R., "Disintegration of the turbulent water jet. J. Hyd. Div. 1, 1964, pp. 175-206.

2. Grant, R.P., Middleman, S., "Newtonian Jet Stability," A. I. Ch. E. J. 12, 1966, pp. 669-678.

3. Phinney, R. E., "The Breakup of a Turbulent Liquid Jet in a Gaseous Atmosphere," J. Fluid Mech. 60, part 4, 1973, pp. 689701.

4. McCarthy, M.J., Malloy, N.A., "Review of Stability of Liquid Jets and the Influence of Nozzle Design," Chem. Eng. J. 7, 1974, pp. 1-20.

5. Wu, P.K., Faeth, G.M., "Onset and End of Drop Formation Along the Surface of Turbulent Liquid Jets in Still Gases," Phys. Fluid A 7, 1995, pp. 2915-2917.

6. Wu P.K., Miranda, R.F, Faeth, G.M., "Effects of Initial Flow Conditions on Primary Breakup of Non-Turbulent and Turbulent Round Jets," At. Sprays Vol. 5, 1995, pp. 175-196.

7. Sallam, K.A., Dai Z., Faeth, G.M, "Liquid Breakup at the Surface of Turbulent Round Liquid Jets in Still Gases," Int. $J$. Multiphase Flow 28, 2002, pp. 427-449.

8. Sallam, K.A., Faeth, G.M., "Surface Properties During Primary Breakup of Turbulent Liquid Jets in Still Air," AlAA Joumal, Vol. 41, 2003, pp.1514-1524.

9. Reitz R.D., "Modeling Atomization Processes in High-Pressure Vaporizing Sprays," Atomization and Spray Technology 3, 1987, pp. 309-337.

10. O'Rourke P. J., Amsden, A. A., "The Tab Method for Numerical Calculation of Spray Droplet Breakup," SAE Technical Paper 872089, 1987.

11. Tanner, F.X., "Liquid Jet Atomization and Drop Breakup Modeling of Non-Evaporation Diesel Fuel Sprays," SAE 1997 Transactions: Journal of Engines, Vol. 106, Sec. 3, 1998, pp. 127-140.

12. Tanner, F.X., “A Cascade Atomization and Drop Breakup Model for the Simulation of High-Pressure Liquid Jets," SAE paper 2003-01-1044.

13. Nishimura, A., Assanis, D. N., “A Model for Primary Atomization Based on Cavitation Bubble Collapse Energy," ICLASS 2000, Pasadena, CA, July 16-20, 2000.

14. Huh K.Y., Lee E., Koo J.Y., "Diesel Spray Atomization Model Considering Nozzle Exit Turbulence Conditions," At. Spray. Vol. 8, 1998, pp. 453-469.

15. Klein, M., Janicka, J., "Large-Eddy Simulation of the Primary Breakup of a Spatially Developing Liquid Film," In ICLASS, Sorrento, Italy, 2003. 
16. De Villiers, E., Gosman, A.D., Weller, H.G., "Large Eddy Simulation of Primary Diesel Spray Atomization," 2004-010100,2004 SAE International.

17. Leboissetier, A., Zaleski, S., "Direct Numerical Simulation of the Atomization of a Liquid Jet," ILASS-Europe, Sept. 2001.

18. Soteriou, C., Andrews, R., Smith, M., "Direct Injection Diesel Sprays and the effect of Cavitation ant Hydraulic Flip Atomization," SAE Paper 950080, 1995.

19. Chaves, H., Knap, M., Kubitzek, A., Obermeler, F., Schneider, T., "Experimental Study of Cavitation in the Nozzle Hole of the Diesel Injectors Using Transparent Nozzles," SAE Paper 950290, 1995.

20. Schmidt, P.D., Rutland J.C., Corradini, L.M., "A Numerical Study of Cavitating Flow through Various Nozzle Shapes," SAE Paper 971597, 1997

21. Trinh, H. P., "Modeling of Turbulence Effect on Liquid Jet Atomization," Ph.D. Dissertation, University of Alabama in Huntsville, 2004.

22. Pope, S.B., Tumbulent Flows, Cambridge University Press, 2000, pp.182-185.

23. Tennekes, H., Lumley, J.L., A First Course in Turbulence, M.I.T. Press., Cambridge, MA, 1972, pp. 248-286.

24. Hiroyasu, H., Kadoła, T., "Fuel Droplet Size Distribution in Diesel Combustion Chamber," SAE Paper 740715, 1974.

25. Yule, A.J., Mo, SL., Tham, S.Y., Aval, S.M., "Diesel Spray Structure," ICLASS-85, Londion, U.K., 1985.

26. Yue, Y., Powell, C., Poola, R., Wang, J., J.K, S., "Quantitative Measurements of Diesel Fuel Spray Characteristics in the Near-Nozzle Region Using X-Ray Absorption," At. Spray, Vol. 11, No. 4, 2001, pp. 471-490.

27. Chou W.H., Hsiang L.P., Faeth, G.M., "Temporal Properties of Drop Breakup in the Shear Breakup Regime," Int. J. Multiphase Flow, Vol. 23, No. 24, 1997, pp. 651-669.

28. Liang, P.Y., Eastes, T.W., Gharakhari, A., "Computer Simulation of Drop Deformation and Drop Breakup," AIAA Paper No. 88-3142.

29. Liu A.B., Mather D., Reitz R.D., "Modeling the Effects of Drop Drag and Breakup on Fuel Sprays," SAE Technical Paper Series 930072, Int. Congress and Exposition, Detroit, Michigan, March 1-5, 1993.

30. Koo, J., "Characteristics of a Transient Diesel Fuel Spray," Ph. D. Dissertation, University of Wisconsin-Madison, 1991.

31. Schneider, B., "Experimental Investigation of Diesel Spray," CRFD and Laser Diagnostic Workshop, $21^{\text {st }}$ CIMAC Congress 1995, Interlaken, Switzerland, May 16, 1995. 